\title{
Beyond the myth of journalistic storytelling: Why a narrative approach to journalism falls short
}

\author{
Guest Editorial Committee \\ Daniel Perrin, ZHAW Zurich University of Applied Sciences, School of Applied Linguistics \\ Marta Zampa, ZHAW Zurich University of Applied Sciences, School of Applied Linguistics* \\ *Corresponding author: marta.zampa@zhaw.ch
}

Despite the journalism education mantra to think story, news media accounts are far more than sheer storytelling. They are more about the punchline first than suspense, more about fragments of information than comprehensive storylines, and more about story-selling than telling. News reporters do not tell stories, as such - but utilize their own narrative routines that evoke stories in people's minds and in the public sphere. This thematic section scrutinizes the widespread storytelling approaches and techniques that journalists are taught and offers fresh and focused insights into narrative practices in the newsroom.

Newswriting is not oriented towards telling entire stories in the sense of classical narrative genres. Instead, it systematically blends linguistic practices of narration and argumentation, of description and explanation, of voicing, inference, enregisterment, intertextuality, and information hierarchy. Stories take an additional life during their appropriation, when the addressees process - or contest - journalists' communicational offers in order to make sense of them. As complex narratives, they become visible only in the follow-up communication and response to news. Social media is a modern-day example of this.

In consequence, public storytelling crucially depends on public sense-making practices as much as on journalists communicating information. From an empirical perspective, research into public storytelling, therefore, aims at explaining why news-making and newswriting practices are likely to evoke this public co-construction of stories. Such analytical insights shed light on practical questions of storytelling. As the authors of the papers in this thematic section argue, in a comprehensive public storytelling framework, the key question for news journalists is not how to tell a story - but how to connect with people's persistent socioculturally reinforced narrative frames in order to be understood.

Gilles Merminod's contribution opens the thematic section by addressing journalistic understanding of the activity of telling a story. Researchers and journalists agree that news has a narrative nature, but what story really means and how a story comes into being in journalistic practice remains unclear. After an overview of existing narrative approaches to news, Merminod argues for a culture-bound definition of story, one that can be discovered only by in-depth ethnographic and linguistic analyses of narrative practices in real newsrooms. This is what he does by analyzing a case study in a French-speaking broadcast newsroom in Switzerland. In this setting, the word histoire (French for story) takes on different meanings - both during the discussion between a journalist and a cutter preparing an item and in the journalist's meta-reflection on his work. At various stages, histoire is used to refer to a genre, a set of information and a semiotic product. Merminod's study helps us understand the use of narrative lexicon in the newsroom and rethink well-established beliefs about the narrative nature of news.

The article by Karl Niklaus Renner enriches the Thematic Section with the perspective of literary studies and its possible applications to journalism research. $\mathrm{He}$ wishes to find out what challenges journalists encounter when telling stories 
that need to fit the criteria of topicality, truthfulness, and objectivity. To this aim, Renner analyzes narrative reporting of the dissertation plagiarism case of the former German Minister of Defense von Guttenberg.

Marta Zampa and Daniel Perrin start from the idea that what journalists believe to be stories are actually incomplete narrative pieces - fragments of narration, to be complemented and completed by the audience during the uptake process. They argue that this incompleteness, besides being related to material limitations, is a rhetorical strategy: leaving already shared information implicit keeps the audience attentive and leads it to agree with the viewpoint expressed in the articles. To provide evidence of the fragmentary nature of journalistic storytelling, Zampa and Perrin analyze the structure of two editorials and two reports in a Swiss Italian-language newspaper. This data is triangulated with results from analyzing journalists' reflection on their writing activity. The results provide evidence that the interplay of narration and argumentation in newswriting as relevant drivers of dynamics and complexity in public discourse deserves precise analyses, which requires interdisciplinary collaboration of production, product, and reception research at the intersection of argumentation and narration theory.

Wibke Weber, Martin Nils Engebretsen and Helen Kennedy deal with a new form of journalism derived from the technological evolution: data journalism. They especially consider whether data visualizations, i.e., "multimodal hybrid artifacts that weave together numbers, words, images and design into a coherent whole" (Weber, Engebretsen \& Kennedy, this volume), count as stories. The authors analyzed 60 interviews with journalists, designers, developers and newsroom leaders from 26 European newsrooms to identify what they believe data stories to be, what characteristics they ascribe to this kind of storytelling, and how storytelling in data journalism can enrich narrative as a research field. Their findings demonstrate that data stories convey a message by showing it, combining the modes of narration, explanation and argumentation, or by enabling users to choose their own story while exploring the data by themselves. Therefore, if we wish to address these new journalistic products as stories, we need to update traditional understandings of stories. 